\title{
Trigeminal Herpes Zoster: A Brief Review
}

\author{
Upender Malik ${ }^{1,{ }^{*}}$, Shilpa Dutta Malik ${ }^{2}$ and Chhavi Srivastava ${ }^{1}$ \\ ${ }^{1}$ Department of Oral Medicine and Radiology, Teerthanker Mahaveer Dental College and Research Centre, 244001, Moradabad, India \\ ${ }^{2}$ Department of Oral Pathology and Microbiology, Teerthanker Mahaveer Dental College and Research Centre, 244001, Moradabad, India \\ "Corresponding author: Department of Oral Medicine and Radiology, Teerthanker Mahaveer Dental College and Research Centre, 244001, Moradabad, India. Email: \\ upender_malik@yahoo.co.in
}

Received 2021 December 19; Revised 2022 January 03; Accepted 2022 January 03.

\begin{abstract}
The reactivated form of the varicella-zoster virus (VZV) is responsible for chickenpox, known as herpes zoster (HZ). Although it is a self-limiting infection, it presents debilitating and painful mucosal and dermal vesicular eruptions. Early identification and management are vital to curbing the spread of $\mathrm{HZ}$ infection. In this extensive review, we present an overview of HZ, including its structure, pathophysiology, clinical presentation, complications, investigations, and management. Our review also highlights the prophylaxis and treatment of complications manifested by the VZV.
\end{abstract}

Keywords: Herpes Zoster, Varicella-Zoster, Postherpetic Neuralgia, Antiviral Therapy, Ramsay Hunt Syndrome

\section{Background}

Herpes zoster (HZ), also known as shingles, derived from the Latin cingulum "girdle", is a secondary manifestation of the varicella-zoster virus (VZV), a ubiquitous DNA virus belonging to the alpha herpes virus group, primarily causing chickenpox which usually occurs in children (1). After resolution of chickenpox, this neurotrophic virus remains latent in the nerve cell bodies and less frequently in the non-neuronal satellite cells of the dorsal root, cranial nerves of which trigeminal nerve is most commonly affected along with C3, T5, L1 \& L2 (2). Intraoral presentation includes unilateral multiple vesicular eruptions that are characteristically dermatomal in distribution. The trigeminal HZ severity and complications carry a high risk of morbidity and mortality, usually implicated in the infection spread and superadded infection, which can be controlled through timely intervention (3).

\section{Pathogenesis}

\subsection{Structure of the Virus}

The VZV belongs to the genus Varicellovirus, family Herpesviridae, subfamily alpha herpesvirinae. It contains an icosadeltahedral virus nucleocapsid, which encloses a single copy of the linear double-stranded DNA genome with a nucleocapsid being surrounded by an assemblage of viral proteins called virion tegument, which is a host-derived lipid envelope further studded with glycoproteins mediating viral attachment, resulting in the initiation of replication upon entry $(4,5)$.

\section{Pathophysiology}

Once transmitted to a susceptible host via aerosolized vesicular content, respiratory droplets, or direct contact with skin lesions, the virus proliferates in the oropharynx and is transported by infected T cells to the skin during an initial viremic phase, resulting in epidermal replication to produce a cell-free VZV. Hence, the characteristic lesions of "varicella" after the local antiviral responses are seen (6).

Varicella is always preceded by a prodrome of 48 - 72 hours, including rash, low-grade fever, malaise, headache, abdominal pain, and anorexia, followed by typical maculopapular rash characterized by "dewdrops on rose petal appearance" filled with fluids, a phase that lasts for 3 - 5 days, followed by 2 - 4 weeks for resolution (7). How the VZV establishes the latency is still debatable. One possibility is that during the viraemic phase, the virus is transported to dorsal or cranial root ganglia within infected $\mathrm{T}$ cells, in which it fuses with neurons, infects neuronal cell bodies, and initiates proliferation. The second possibility is the transfer of the virus to cell bodies via retrograde axonal transport once cell free varicella zoster infects the intraepidermal projections of sensory neurons after it is produced in the epidermis (8). This latency can be found in ganglia like geniculate, vestibular, trigeminal, cervical, 
thoracic, and sacral ganglia. Trigeminal ganglia are most commonly affected in the head and neck regions until the VZV gets reactivated and causes $\mathrm{HZ}$ years after the initial infection (in the older age group) once the immunity has waned or there are other predisposing factors including physical trauma, increased age, stress, radiation therapy, and immunocompromised states like HIV infection $(3,9)$.

Secondary manifestations of $\mathrm{HZ}$ are characterized by unilateral vesicular eruptions uniformly followed by a prodrome of deep, aching, or burning pain with little to no fever or lymphadenopathy (6).

\section{Clinical Features}

Varicella is one of the mildest infections affecting children below 10 years of age, although it can occur at any age $(7,10)$. The lesion begins as pruritic rashes, including maculopapular/pustular eruptions on an erythematous base with various stages of evolution that begins on the trunk and face, which is the hallmark of infection and spreads centrifugally to involve other body areas. It is succeeded by crops appearing over 2 - 4 days, always preceded by a prodrome of low-grade fever, malaise, headache, and vomiting (7). The lesion severity is $2-3$ folds higher in immunocompromised patients as they are at greater risk of visceral complications, which have been reported to be fatal in $15 \%$ of cases with secondary bacterial superinfection of the skin as the most common infection complications along with others like myocarditis, pneumonia, and hepatitis (7).

On the other hand, HZ, also known as shingles, is a sporadic disease resulting from VZV reactivation, which becomes latent in cranial/dorsal root ganglia in individuals primarily affected by varicella in the past. It occurs at all ages with an incidence of 5 - 10 cases per 1,000 persons aged above 60 years of age (7). The lesion typically is limited to one or two dermatomes with thoracic dermatome (50 - 60\%), cervical dermatome (10 - 20\% and trigeminal dermatome), being frequently involved with lumbar and sacral dermatomes, showing the least involvement, presented as unilateral rashes on the face and trunk, and sometimes involving extremities that further develop crustations and resolve in about two weeks $(3,11)$.

Secondary manifestations of HZ show dermatomal distribution due to the trigeminal nerve involvement (3). Involvement of ophthalmic division leads to lesions on the forehead, scalp, and upper eyelid, which may lead to acute retinal necrosis, lesions on midface, and maxillary division where the patient experiences a prodrome of pain and burning sensation with tenderness on the palate/gingiva unilaterally, followed by painful clustered ulcers of 1-5 mm in size whereas the involvement of mandibular division results in ulcerations on the tongue and mandibular gingiva. In immunocompromised patients, vesicular eruptions are more persistent on oral soft tissues and have a higher tendency of extending to the underlying bone, causing tooth exfoliation, osteonecrosis, and vitiligo (1).

\section{Complications}

The incidence of $\mathrm{HZ}$ complications increases with age and can be divided into four groups: Cutaneous, visceral, neurological, and ocular, with postherpetic neuralgia being most common, followed by ocular complications (8). Postherpetic neuralgia is a potential sequela of $\mathrm{HZ}$ resulting from inflammatory/viral damage to the components of the primarily affected sensory pathway. It is a debilitating condition where the pain of postherpetic neuralgia begins in the area of healed rashes within three months. Pain due to a stimulus is inordinate to it, accompanied by allodynia and other abnormal sensations like paresthesia or dysesthesia in individuals above 50 years of age. The ophthalmic branch is most commonly affected when the trigeminal nerve is involved, resulting in $\mathrm{HZ}$ ophthalmicus due to the innervation of VZV in gasserian ganglion, including a nasociliary branch, which may present ocular damage ranging from conjunctivitis to retinal necrosis (6).

Ramsay hunt syndrome is a neurological complication of HZ due to the involvement of geniculate ganglion of the facial nerve and is presented as vesicles on and palate associated with otalgia, tinnitus, deafness, and loss of taste from the anterior two-thirds of the tongue as a result of the involvement of chorda tympani branch of the facial nerve $(1,12)$.

Herpes zoster encephalitis is a rare neurological complication that can occur before, simultaneously, or after the rash, affecting the immunocompromised elderly with headaches, fever, mental status changes, and seizures as clinical presentations, usually subacute (13).

Zoster sine herpete is well known as chronic radicular pain without rash caused by VZV reactivation with typical dermatomal distribution, which is a rare occurrence and can be studied virologically to confirm VZV reactivation (14). Herpes zoster in children can occur in newborns within a few weeks after birth, with thoracic dermatome being most commonly involved and cranial sites rarely affected. Affected children have a history of intrauterine exposure to the VZV or exposure until four years of age or are immunosuppressed. The clinical presentations of vesicular lesions of $\mathrm{HZ}$ affecting children of this age group are often misdiagnosed as impetigo or other cutaneous disorders (15). 
Zoster paresis can occur as a result of VZV reactivation from ganglia in cervical, thoracic, or lumbosacral regions where arm weakness or diaphragmatic paralysis can occur in cervical distribution zoster, abdominal muscle weakness, and hernia in thoracic distribution zoster, whereas leg weakness in lumbosacral distribution zoster (16).

\section{Investigations}

Serum immunoglobulin/antibodies are glycoproteins produced by plasma cells acting as an essential part of immune response, which recognizes and binds to specific antigens such as bacteria, virus, fungi, or parasites, aiding in their destruction (17). After initial exposure to the VZV, serum IgM titer is found to be raised soon after the appearance of vesicles, i.e., within 2 - 3 days, marking the acutestage infection, which reaches its maximum titer after 8 - 11 days, followed by a rapid decline to the point of being undetectable after a year. In convalescent person, presence of serum IgM marks an acute stage of the disease which tends to decline, whereas IgG titers appear to persist after varicella and these IgG titer rises to 4 folds (approx.) at the time of secondary exposure (17).

Histologic features of herpetic vesicles show intraepithelial blisters containing fluids. The virus-infected cells portray acantholysis with the formation of Tzank cells with large vesicular nuclei comprising pale eosinophilic cytoplasm, termed ballooning degeneration. Intracellularly, chromatin condensation with nucleolar fragmentation around the periphery of the nucleus is seen along with fusions between adjacent cells forming infected epithelial cells. When the vesicles rupture, exudates like fibrin, PMNLs, and degenerated cells cover the tissue surface, and these lesions are healed by peripheral epithelial proliferation (18).

The TORCH test, also known as the TORCH panel, is an acronym for toxoplasmosis, rubella, and cytomegalovirus and includes other infections like herpes simplex varicellazoster and syphilis. This test screens for the presence of IgM and IgG antibodies during pregnancy to determine the immune status of the mother where if IgG antibodies are present at a specific concentration, then it indicates immunity against Toxoplasma gondii, rubella, cytomegalovirus, and herpes simplex virus, ruling out infection transmission to the fetus (19).

Cell culture is highly specific and performed by inoculating fluids aspirated from vesicles, then incubating at $36^{\circ} \mathrm{C}$ in a $5 \% \mathrm{CO}_{2}$ atmosphere and holding for a maximum of 14 days before declaring negative. The process can be catalyzed by the centrifugation of cell cultures in shell vials, and the identification is confirmed by staining the monolayer of lung cells or human fetal diploid kidney on which the vesicular fluid is inoculated with VZV-specific monoclonal bodies (20).

A more specific diagnosis can be made by immunohistochemical staining to detect VZV glycoproteins in infected epithelial cells using fluorescein-binding monoclonal antibodies. This allows for rapid diagnosis in the case of atypical clinical symptoms (20). Polymerized chain reaction is an important diagnostic method for detecting VZV DNA in the CSF of VZV encephalitis patients, ocular fluids, and VZV retinitis tissues. It also overcomes the complications of culturing labile VZV (20).

\section{Treatment}

Primary considerations in the treatment of HZ infection cover the treatment of acute-phase infection, prophylactic management, and managing complications associated with $\mathrm{HZ}$ infections.

\subsection{Antiviral Therapy}

Antiviral therapy of AZV aims to manage infections by decreasing pain and promoting healing if initiated within 48 - 72 hours of rash onset.3 Acyclovir is an acyclic guanine nucleoside analog that acts by phosphorylation to a monophosphate derivate by viral-specific thymidine kinase, which is further phosphorylated to ACV triphosphate by cellular kinase to inhibit viral DNA synthesis (21). It is given orally $800 \mathrm{mg}$ five times a day for 7 - 10 days. It has a bioavailability of 15 - 30\% and a half-life of three hours. When given within 72 hours of rash, it decreases pain and promotes healing, but the intensity of postherpetic neuralgia is not reduced $(3,22)$. The drug is well tolerated orally, but some cases of headache, nausea, malaise, and CNS effects have been reported, whereas rashes, sweating, hypotension, and emesis when given intravenously (21).

Valacyclovir, a prodrug of acyclovir, when administered orally, overcomes the problem of poor bioavailability of acyclovir. It is given orally $1000 \mathrm{mg}$ thrice daily for 7-10 days, resulting in quicker healing to reduce the incidence of postherpetic neuralgia $(3,23)$.

Famciclovir, a prodrug of penciclovir, has good oral bioavailability (77\%) given $500 \mathrm{mg}$ thrice a day for 7-10 days for patients with uncomplicated HZ, whereas dose adjustment is required in patients with creatinine clearance of $<$ $60 \mathrm{~mL} / \mathrm{min}(21,23)$.

Foscarnet, a pyrophosphate analog, is administered 40 $\mathrm{mg} / \mathrm{kg}$ IV every eight hours in immunocompromised patients with acyclovir-resistant VZV until the lesion healing occurs. The most common adverse effect due to foscarnet therapy is nephrotoxicity $(3,24)$.

Though contraindicated in immunocompromised patients, Corticosteroids can be administered to the elderly 
in combination with antiviral drugs. Methylprednisolone is most commonly given as a single dose of up to $60 \mathrm{mg}$ per day orally with antivirals, showing beneficial pain resolution, faster lesion healing, and quality of life improvement $(3,24)$.

Interferons are species-specific cellular glycoproteins produced naturally by virus-infected cells with antiviral protective action. It inhibits the protein synthesis of the virus by inducing new cellular RNA and protein production after binding to specific cell surface receptors. They also act as immunomodulators, thus increasing cytotoxic T lymphocyte activity, amplifying NK cells activity, and activating macrophages. They do not penetrate the CSF and predominantly undergo proteolytic degradation in renal tubules. The administration of $\alpha$-IFN in immunocompromised patients with $\mathrm{HZ}$ reduces the risk of viral dissemination but does not promote rash healing (25).

\subsection{Prophylaxis}

Vaccination has proven to be a proactive strategy in reducing $\mathrm{HZ}$ and postherpetic neuralgia. Takahashi and his colleagues developed a live attenuated vaccine from the Oka strain of VZV isolated from a healthy child with varicella and attenuated in human and guinea pigs.28 As per ACIP recommendations, the live attenuated vaccine can be administered as a single dose subcutaneously in immunocompetent individuals or those having a history of $\mathrm{HZ}$, which is effective for a maximum of eight years. It is contraindicated in patients on immunosuppressive therapy with untreated TB, primary and acquired immunodeficiency, and pregnancy $(26,27)$. The recombinant, adjuvanted zoster vaccine contains glycoprotein E of VZV, which plays an essential role in replication and cell-to-cell spread, a target of specific response along with AS01B adjuvant that initiates innate immune response activation (26, 27).

\section{Management of Complications}

Postherpetic neuralgia is the most common complication of HZ, which is challenging to manage and often resistant to pharmacological approaches. Therefore, a multimodal analgesic treatment to achieve effectiveness and acceptability of the therapeutic regimen should be performed (23).

Treatment can be done both pharmacologically and non-pharmacologically (23). Pharmacological management consists of applying topical agents of anesthetics and analgesics locally to relieve the pain (23). Besides, 5\% lidocaine patch application as a topical agent produces significant pain relief in postherpetic neuralgia and allodynia.
A maximum of three patches can be applied for 12 hours a day over the affected area, and its efficacy is noted after 7 10 days of treatment $(23,24)$. Capsaicin, an alkaloid and an active neuropeptide agent derived from hot chili peppers, can be applied at concentrations of 0.025 and $0.075 \%$ as a topical agent $(22,23)$. Also, PHN, a typical complication of $\mathrm{HZ}$, can be managed systemically by three classes of medication: Anticonvulsants, antidepressants, and opioid analgesics.

Anticonvulsants like gabapentin and pregabalin have proven to be safe and well-tolerated in reducing pain associated with $\mathrm{HZ}(23,24)$. Gabapentin, initiated at $300 \mathrm{mg}$ per day and increasing up to $3600 \mathrm{mg}$ per day as required for pain regulation given to patients, shows superior effects in relieving pain in PHN patients. It is absorbed slowly and reaches a peak at 3 - 4 hours post-administration, and has frequent adverse effects like somnolence, dizziness, and peripheral edema. In contrast, pregabalin, a potent gabapentinoid and structural analog of GABA is an ion channel modulator with rapid analgesic actions and fewer side effects. Depending on effectiveness and tolerability, pregabalin can be given 100 - $150 \mathrm{mg}$ per day as a starting dose as a starting dose to a maximum of $600 \mathrm{mg}$ per day (22). Tricyclic antidepressants like amitriptyline, nortriptyline, and desipramine inhibit spiral neurons involved in nociceptive perception by blocking the neuronal uptake of noradrenaline and serotonin (22).

Opioid analgesics also alleviate the PHN pain by binding to opioid receptors such as $\mu, \mathrm{K}, \delta$, and nociceptors present in the central and peripheral nervous system but cannot be used as the first-line treatment due to tolerance and concerns related to its misuse. Opioids have specific side effects like cardiorespiratory dysfunction, sedation, nausea/vomiting, histamine release, and constipation as significant side effects in elderly patients (22).

Non-pharmacological pain management methods like nerve blocks, TENS, and botulinum toxin can be used in patients not relieved from pharmacological approaches. Sympathetic nerve blocks can be used in treating patients with acute $\mathrm{HZ}(22,24)$. Besides, TENS is an adjunctive therapy that transiently relieves pain utilizing electric currents produced by a device to stimulate nerves at a vigorous intensity $(22,24)$. Botulinum toxin-A also plays an adjunctive role in pain relief in PHN patients with proven efficacy, tolerability, and safety along with inducing antitoxin antibodies $(22,24)$.

Treatment of herpes zoster opthalmicus (HZO) with oral antivirals within 72 hours of symptom onset helps reduce the severity of the condition and the long-term complications. The recommended treatment is oral acyclovir $800 \mathrm{mg}$ five times a day for 7-14 days regardless of the stage of $\operatorname{HZO}(28,29)$. 
Ramsay hunt syndrome shows a significant response to antiviral therapy with steroids. Oral corticosteroids such as prednisolone $60 \mathrm{mg} /$ day as initial doses can be prescribed with a 10-day tapering course combined with either acyclovir or valacyclovir. Temporary relief from otalgia can be achieved by applying local anesthetics or cocaine if the trigger point is in the external auditory canal. Inability to close eyes in individuals with Ramsay hunt syndrome can expose the cornea to abnormal drying and foreign body irritation, which can be managed using artificial tears and eyepatches. The patches used should be with the otolaryngologist consultation for changes in hearing (30).

\section{Conclusion}

The varicella-zoster virus has a remarkable ability to remain latent for years and reappear as $\mathrm{HZ}$ to affect an individual's life. An oral physician can be the first one to identify the signs of the viral infection and treat it to prevent its propagation. Therefore, thorough knowledge and understanding of the subject are essential. Moreover, further research still demands attention to the arena of vaccine development.

\section{Footnotes}

Authors' Contribution: Study concept and design, Upender Malik; Acquisition of data and drafting of the manuscript, Chhavi Srivastava; Critical revision of the manuscript for important intellectual content, Shilpa Dutta.

\section{Conflict of Interests: There is no conflict of interest.} Funding/Support: There is no funding/support.

\section{References}

1. Rai NP, Chattopadhyay J, Abhinandan SG. Herpes Zoster Infection of Maxillary and Mandibular Branch: A Case Report and Current Trends in Management. Int J Contemp Med Res. 2016;3(5):1437-40.

2. Babamahmoodi F, Alikhani A, Ahangarkani F, Delavarian L, Barani H, Babamahmoodi A. Clinical manifestations of herpes zoster, its comorbidities, and its complications in north of iran from 2007 to 2013. Neurol Res Int. 2015;2015:896098. doi: 10.1155/2015/896098. [PubMed: 25893116]. [PubMed Central: PMC4393919].

3. Malik U, Sunil M, Gupta C, Kumari M. Trigeminal Herpes Zoster: Early Recognition and Treatment -A Case Report. Journal of Indian Academy of Oral Medicine and Radiology. 2017;29(4):350. doi: 10.4103/jiaomr.jiaomr_102_17.

4. Zerboni L, Arvin AM. The pathogenesis of varicella-zoster virus neurotropism and infection. Neurotropic viral infections. New York, USA: Springer; 2016. p. 135-73.

5. Warren-Gash C, Forbes H, Breuer J. Varicella and herpes zoster vaccine development: lessons learned. Expert Rev Vaccines. 2017;16(12):1191-201. doi: 10.1080/14760584.2017.1394843. [PubMed: 29047317]. [PubMed Central: PMC5942150].
6. Makos C, Nousios G, Peios M, Evangelinou C, Balabanis G. Herpes zoster of the trigeminal nerve-two cases reports. Thessaloniki, Greece: Aristotle University of Thessaloniki; 2011.

7. Whitley RJ. Varicella-Zoster Virus Infections. In: Kasper D, Fauci A, Hauser S, Longo D, Jameson J, Loscalzo J, editors. Harrison's principles of internal medicine. 16th ed. New York, USA: Mcgraw-Hill; 2005.

8. Gershon AA, Gershon MD, Breuer J, Levin MJ, Oaklander AL, Griffiths PD. Advances in the understanding of the pathogenesis and epidemiology of herpes zoster. J Clin Virol. 2010;48(Suppl 1):S2-7. doi: 10.1016/S1386-6532(10)70002-0. [PubMed: 20510263]. [PubMed Central: PMC5391040].

9. Prest VT, Melo RB, Sá CDL, Carneiro NCM, Bernardino RDS, Feitosa VP. Clinical report of rare manifestation of Herpes Zoster in the mandibular nerve. Res Soc Dev. 2021;10(4). e46010414225. doi: 10.33448/rsd-v10i4.14225.

10. Andrei G, Snoeck R. Advances and Perspectives in the Management of Varicella-Zoster Virus Infections. Molecules. 2021;26(4). doi: 10.3390/molecules26041132. [PubMed: 33672709]. [PubMed Central: PMC7924330].

11. Blair RJ. Varicella Zoster Virus. Pediatr Rev. 2019;40(7):375-7. doi: 10.1542/pir.2017-0242. [PubMed: 31263049].

12. Wehrhahn MC, Dwyer DE. Herpes zoster: epidemiology, clinical features, treatment and prevention. Australian Prescriber. 2012;35(5):1437. doi: 10.18773/austprescr.2012.067.

13. Kennedy PGE, Gershon AA. Clinical Features of Varicella-Zoster Virus Infection. Viruses. 2018;10(11). doi: 10.3390/v10110609. [PubMed: 30400213]. [PubMed Central: PMC6266119].

14. Mueller NH, Gilden DH, Cohrs RJ, Mahalingam R, Nagel MA. Varicella zoster virus infection: clinical features, molecular pathogenesis of disease, and latency. Neurol Clin. 2008;26(3):675-97. viii. doi: 10.1016/j.ncl.2008.03.011. [PubMed: 18657721]. [PubMed Central: PMC2754837].

15. Gross G. Clinical Picture and Complications of Herpes Zoster: The View of the Dermatologist. In: Gross G, Doerr H, editors. Herpes zoster : recent aspects of diagnosis and control. Basel, Switzerland: Karger; 2006. p. 20-36. doi: 10.1159/000096256.

16. Nagel MA, Gilden D. Complications of varicella zoster virus reactivation. Curr Treat Options Neurol. 2013;15(4):439-53. doi: 10.1007/s11940013-0246-5. [PubMed: 23794213]. [PubMed Central: PMC3752706].

17. Brunell PA, Gershon AA, Uduman SA, Steinberg S. Varicella-Zoster Immunoglobulins during Varicella, Latency, and Zoster. J Infect Dis. 1975;132(1):49-54. doi:10.1093/infdis/132.1.49. [PubMed: 169308].

18. Regezi JA, Sciubba JJ, Jordan RCK. vesiculobullous diseases. Oral Pathology: Clinical Pathologic Correlations. 4th ed. Amsterdam: Elsevier Health Sciences; 2003.

19. Fuchs FE, Pauly M, Black AP, Hubschen JM. Seroprevalence of ToRCH Pathogens in Southeast Asia. Microorganisms. 2021;9(3). doi: 10.3390/microorganisms9030574. [PubMed: 33799699]. [PubMed Central: PMC7999562].

20. Schmader KE, Oxman MN. Varicella and Herpes Zoster. In: Goldsmith LA, Katz SI, Gilchrest BA, Paller AS, Leffell DJ, Wolff K, editors. Fitzpatrick's Dermatology in General Medicine. 8th ed. New York, USA: McGraw Hill Medical; 2012. p. 2421-9.

21. Tripathi KD. Antifungal and Antiviral drugs. Essentials of Pharmacology for Dentistry. 2nd ed. Noida, India: Jaypee; 2016.

22. Jung HY, Park HJ. Pain Management of Herpes Zoster. In: Prostran M, editor. Pain Management. London, UK: IntechOpen; 2016. doi: $10.5772 / 62873$.

23. Saguil A, Kane S, Mercado M, Lauters R. Herpes Zoster and Postherpetic Neuralgia: Prevention and Management. Am Fam Physician. 2017;96(10):656-63. [PubMed: 29431387].

24. Koshy E, Mengting L, Kumar H, Jianbo W. Epidemiology, treatment and prevention of herpes zoster: A comprehensive review. Indian JDermatol Venereol Leprol. 2018;84(3):251-62.doi:10.4103/ijdvl.IJDVL_1021_16. [PubMed: 29516900]. 
25. Satoskar RS, Rege N, Bhandarkar SD. Pharmacology and Pharmacotherapeutics. 24th ed. Amsterdam: Elsevier Health Sciences; 2015.

26. Harbecke R, Cohen JI, Oxman MN. Herpes Zoster Vaccines. J Infect Dis. 2021;224(12 Suppl 2):S429-42. doi: 10.1093/infdis/jiab387. [PubMed: 34590136]. [PubMed Central: PMC8482024].

27. Gabutti G, Bolognesi N, Sandri F, Florescu C, Stefanati A. Varicella zoster virus vaccines: an update. Immunotargets Ther. 2019;8:1528. doi: 10.2147/ITT.S176383. [PubMed: 31497569]. [PubMed Central: PMC6689529].

28. Dworkin RH, Johnson RW, Breuer J, Gnann JW, Levin MJ, Backonja
$\mathrm{M}$, et al. Recommendations for the management of herpes zoster. Clin Infect Dis. 2007;44(Suppl 1):S1-26. doi: 10.1086/510206. [PubMed: 17143845].

29. Rousseau A, Bourcier T, Colin J, Labetoulle M. Herpes Zoster Ophthalmicus-Diagnosis and Management. US Ophthalmic Rev. 2013;6(2).

30. Ghofran MH, Yousif IE, Ahmed A, Abusofyan S, Ahmed MS. Ramsay Hunt Syndrome: Case Presentation and Management. Adv Dent $\&$ Oral Health. 2020;12(2):555835. doi: 10.19080/adoh.2019.11.555835. 